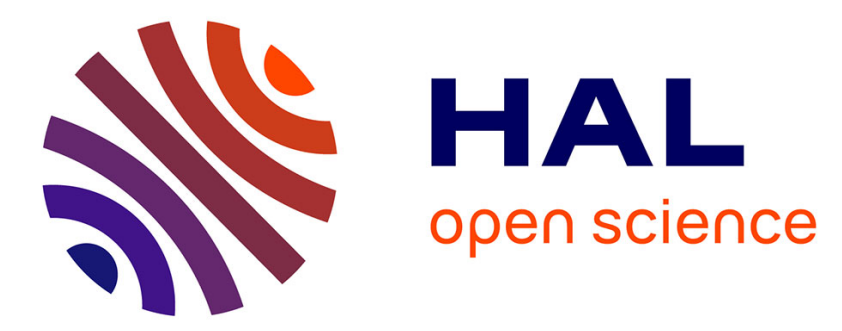

\title{
Plasmonic Nano-Objects: From Subwavelength Field Enhancement to Hot Charges and Luminescence
}

Céline Molinaro, Sylvie Marguet, Ludovic Douillard, Fabrice Charra, Céline Fiorini-Debuisschert

\section{- To cite this version:}

Céline Molinaro, Sylvie Marguet, Ludovic Douillard, Fabrice Charra, Céline Fiorini-Debuisschert. Plasmonic Nano-Objects: From Subwavelength Field Enhancement to Hot Charges and Luminescence. 2019 21st International Conference on Transparent Optical Networks (ICTON), Jul 2019, Angers, France. pp.1-4, 10.1109/ICTON.2019.8840397 • cea-02389187

\section{HAL Id: cea-02389187 https://hal-cea.archives-ouvertes.fr/cea-02389187}

Submitted on 2 Dec 2019

HAL is a multi-disciplinary open access archive for the deposit and dissemination of scientific research documents, whether they are published or not. The documents may come from teaching and research institutions in France or abroad, or from public or private research centers.
L'archive ouverte pluridisciplinaire HAL, est destinée au dépôt et à la diffusion de documents scientifiques de niveau recherche, publiés ou non, émanant des établissements d'enseignement et de recherche français ou étrangers, des laboratoires publics ou privés. 


\title{
Plasmonic Nano-Objects: From Subwavelength Field Enhancement to Hot Charges and Luminescence
}

\author{
C. Molinaro ${ }^{1}$, S. Marguet ${ }^{2}$, L. Douillard ${ }^{1}$, F. Charra $^{1}$, and C. Fiorini-Debuisschert ${ }^{1}$ \\ 1. SPEC, CEA, CNRS, Université Paris-Saclay, 91191 Gif-sur-Yvette cedex, France \\ 2. NIMBE, CEA, CNRS, Université Paris-Saclay, 91191 Gif-sur-Yvette, France \\ Tel: +33(0) 1690862 38, e-mail: celine.fiorini@cea.fr
}

\begin{abstract}
Although bulk gold is known to present very low luminescence quantum yield, gold nanoparticles can present a huge nonlinear luminescence with an apparent high influence of the particle shape. The two-photon luminescence (TPL) of Au nanorods (NR) was investigated at the single-object level, combining polarizationresolved TPL and simultaneously acquired topography using atomic force microscopy (AFM). We evidence that the huge TPL observed in these rods is the result of an interplay between their two different localized surface plasmon resonances. Excitation of gold NR at its longitudinal plasmon frequency first lead to increased absorption and thus increased production of electron-hole pairs that happen to recombine radiatively through the transverse plasmon band i.e. with an enhanced emission at the NR transverse plasmon band. Above fundamental aspects, we show that these specific properties make TPL a highly sensitive technique for the characterization of nanoobjects from their topology to their organization.
\end{abstract}

Keywords: gold nanoparticles, plasmons, two-photon luminescence, hot charges.

\section{INTRODUCTION}

Nano-objects of noble metals exhibit collective oscillation modes of their conduction electrons known as local plasmons. Beyond spectacular colors, plasmon modes can induce subwavelength electromagnetic field confinement [1,2] associated to field enhancement effects which are of major interest towards future applications, from selective photochemistry or phototherapy to new multifunctional characterization techniques such as surface or tip-enhanced microscopies and spectroscopies [3].

In addition to these nano-antenna properties, plasmonic nanoobjects, and gold particles more particularly, were shown to exhibit quite unexpected luminescence properties, considering the very low quantum yield of bulk gold [4]. Plasmonic luminescence has given rise to many studies but continues to be the subject of many discussions. Different origins have been proposed from inelastic light scattering to multiphoton luminescence or even thermal radiation [5]. One difficulty comes from the differences in the excitation conditions from one reported experiment to another, from e.g. continuous to pulsed excitation, or average to single particle measurements. The gold band structure or the crystalline properties of the considered nano-objects might also play an important role.

We report here detailed investigations of the two-photon luminescence (TPL) properties of colloidal gold nanorods (GNR) studied at the single particle level to prevent any effect of sample heterogeneity. The size and aspect ratio of the nanorods were chosen so as to exhibit a longitudinal plasmon resonance around $800 \mathrm{~nm}$, for nonlinear excitation using a Ti-Sapphire femtosecond laser source as further detailed below.

An in-depth study of the influence of both the wavelength and polarization of the excitation beam was carried out. We demonstrate that the nanorods TPL results from two subsequent steps involving both the longitudinal and the transverse surface plasmon resonances of the nanorods: an increase of electron-hole generation first results from local electric field enhancement at the longitudinal plasmon resonance; the relaxation of electronhole pairs through the excitation of the transverse plasmon mode of the rod then plays an important role [6]. In a next step, the influence of incident power was investigated using the conditions (polarization and wavelength) within which the maximum TPL could be obtained. We show that TPL reveals itself as a highly sensitive tool to follow tiny changes resulting from the photo-induced reshaping of nanorods [7], the high anisotropic character of TPL making it also an interesting way to characterize the organization of nanorods [8].

\section{SAMPLE PREPARATION}

40-nm long, 10-nm diameter cylindrical GNRs were synthesized using a now well-established colloidal seedmediated procedure $[9,10]$. Transmission electron microscopy (TEM) characterizations reveal the perfect, single crystalline nature of these GNRs which extinction spectrum is shown in Fig. 1a. These GNRs exhibit two different plasmon resonances: a transverse resonance $\lambda^{\mathrm{T}} \sim 520 \mathrm{~nm}$ and a longitudinal one $\lambda^{\mathrm{L}} \sim 800 \mathrm{~nm}$ as expected from their aspect ratio (AR) of c.a. 4 [11] and further confirmed following simulations using the Boundary Element Method (BEM) [12]. BEM was also used to calculate the field map expected for these $10 \mathrm{~nm}$ $\times 40 \mathrm{~nm}$ rods. As shown in Fig. 1b, it reveals very high field enhancements at both the wavelengths of the transverse and, to a larger extent, the longitudinal plasmon resonances. 


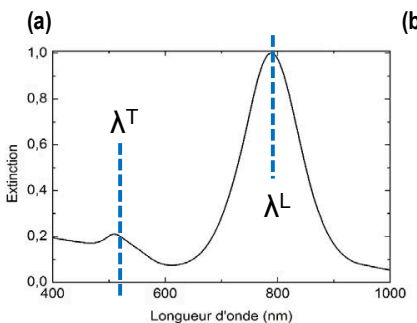

(b)

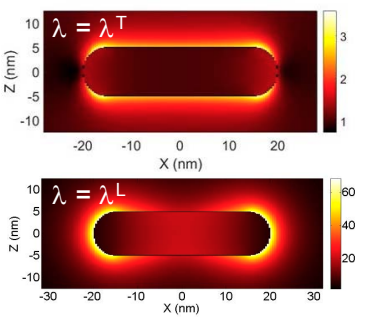

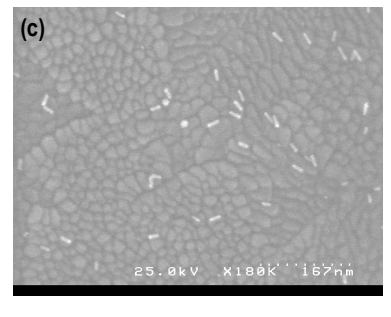

Figure 1: (a) Extinction spectrum of a solution of $10 \mathrm{~nm} \times 40 \mathrm{~nm}$ GNR dispersed in water; (b) Field enhancement maps calculated using BEM, considering excitation of either the transverse or longitudinal GNR plasmon resonances; (c) SEM image of nanorods dispersed onto an ITO coated glass substrate.

Given the colloidal synthesis method, a positively charged CTAB (Cetyl TrimethylAmmonium Bromide) capping layer surrounds the nanorods $[9,10]$. The positive charge of the CTAB surfactant is taken into profit to immobilize the GNR using electrostatic interactions. For this, ITO coated substrates are first treated using UVOzone in order to produce negatively charged hydroxyl groups at the ITO surface, a droplet of GNRs in solution being consecutively left to dry. Non-specifically coupled GNRs are then eliminated through careful rinsing with ethanol. Thanks to specific landmarks fabricated on the substrates, each considered GNR can be precisely localized and the same single objects can be analyzed using either TPL, AFM and scanning electron microscopy (SEM, as shown in Fig. 1c).

\section{CORRELATED TPL AND TOPOGRAPHY ANALYSES OF SINGLE NANORODS}

The two-photon luminescence (TPL) of single $10 \mathrm{~nm} \times 40 \mathrm{~nm}$ colloidal gold nanorods was characterized using a specific set-up developed in order to correlate each GNR TPL properties with its geometry, orientation and environment (see Fig. 2a). Each GNR is simultaneously characterized using two probes that are previously aligned: (1) an AFM tip for the characterization in topography (NanoWizard III, JPK), and (2) a focused laser spot (microscope objective NA $=1.3$ leading to a beam waist $w=400 \mathrm{~nm}$ ) coupled to the sample through an inverted optical configuration, for the two-photon excitation of the nanorods (Tsunami, Spectra Physics $740 \mathrm{~nm}<\lambda<950 \mathrm{~nm}$ ). The same objective is also used for collection of the luminescence which is further analysed using either a channel plate multiplier working in the photon counting mode (Perkin Elmer MP-993$C L$ ) or a spectrometer coupled to a CCD camera (Andor DU401-BR-DD) as further discussed below.
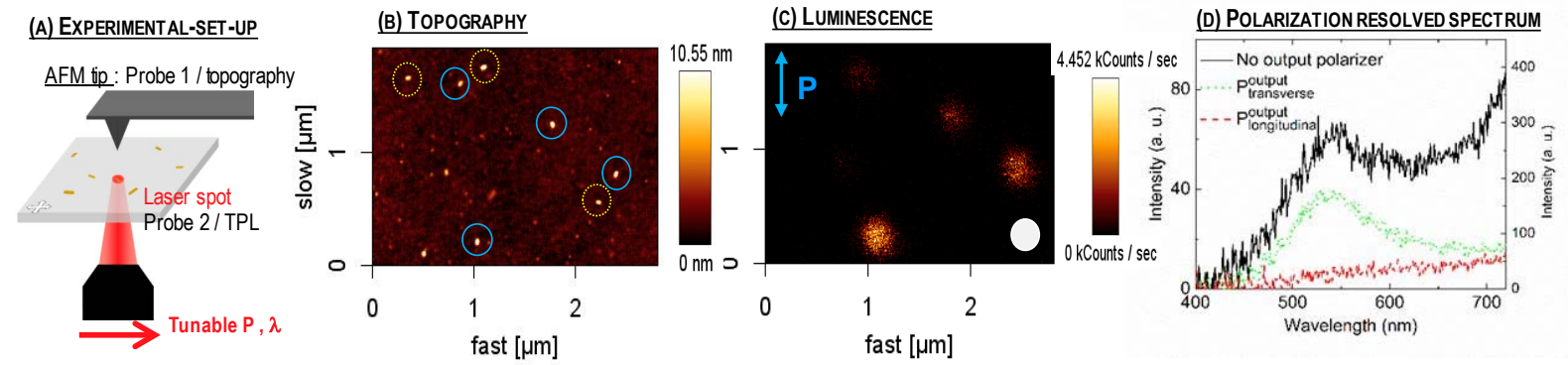

Figure 2: (a) simplified scheme of the combined TPL and AFM microscopy set-up; (b and c) Simultaneous topography and TPL signal measured on a sample of GNRs previously immobilized onto an ITO covered glass substrate. The orientation of each GNR is distinguishable even if a slight broadening of the GNRs is observed due to the AFM tip convolution with the rods, a TPL signal being only observed for the rods which longitudinal axis is closed to the exciting polarization direction. (d) Polarization resolved analysis of the spectrum of a single GNR TPL emission. The GNR was excited at its resonance wavelength with an incident power of $20 \mu \mathrm{W}$, and an exciting beam polarization along its longitudinal axis (for maximum TPL).

As evidenced in Fig. 2, the TPL signal intensity appears to be highly dependent on the GNRs orientation for a given incoming laser beam polarization. Detailed angular dependence studies have been realized on different single GNRs with different spatial orientations, showing more particularly that the TPL intensity varies as $\cos ^{4}(\theta)$, with $\theta$ the angle between the laser polarization and the rod longitudinal axis [6]. This dependence which could be reproduced on all considered GNRs, indicates not only the dipolar origin of the signal but evidences also the quadratic dependence of the signal with the excitation beam. The correlation between the topography measurements and the TPL intensity points out that the angle of maximum luminescence of a GNR is observed each time in the direction of its longitudinal axis.

The influence of the exciting beam wavelength $\lambda_{\text {exc }}$ was further studied using a polarization along the longitudinal axis of the GNR: the maximum TPL is obtained at the GNR longitudinal surface plasmon resonance 
$\left(\lambda_{\text {exc }}=\lambda^{\mathrm{L}}\right)$, in agreement with previously reported studies [13]. The TPL data measured for a large number of single GNRs appear moreover to be very well described (no external adjustable parameters) by the theoretical dependence that can obtained from the BEM simulations or from the analytical Gans-Mie model [6].

As a complementary characterization, the spectral content of the TPL signal was also analyzed through a spectrometer coupled to a CCD camera. As shown in Fig. 2d, two emission bands are evidenced, varying both quadratically with the laser excitation power and peaking, one in the IR and the second around $\lambda_{\mathrm{TPL}}{ }^{\text {max, VIS }}=547$ $\pm 4 \mathrm{~nm}$, i.e. very close to the GNRs transverse surface plasmon band. This band appears moreover to be $80 \%$ polarized along the GNR transverse axis, i.e. in a direction perpendicular to the exciting beam polarization.

From this, we conclude that the large two-photon luminescence in gold nanorods results from the interplay of their two different plasmon resonances (transverse, $\lambda^{\mathrm{T}}$, and longitudinal, $\lambda^{\mathrm{L}}$, plasmon modes) : excitation at the GNR longitudinal plasmon resonance $\left(\lambda_{\text {exc }} \approx \lambda^{\mathrm{L}}\right)$ results in increased absorption, obviously leading to increased production of electron-holes pairs. These electron-hole pairs subsequently recombine radiatively through the GNR transverse plasmon band $\left(\lambda_{\mathrm{em}} \approx \lambda^{\mathrm{T}}\right)$ leading to plasmon enhanced emission [6,14]. Direct radiative relaxation of electron and holes can also be observed [15], but in a much less extent, which explains the fact that visible TPL is not fully polarized ( $80 \%$ degree of polarization).

\section{NP TOPOLOGY OR ORGANIZATION}

As discussed above and illustrated in Fig. 2, TPL is highly dependent on the GNR orientation, varying as $\cos ^{4}(\theta)$, with $\theta$ the angle between the incident light polarization and the GNR longitudinal axis. TPL characterization can thus interestingly be used in order to study nanorods arrangements.

Considering liquid crystalline (LC) matrices, which are well known to be intrinsically anisotropic, we could evidence TPL images fundamentally different when going from an incident laser polarization parallel to perpendicular to so-called LC oily streaks (smectic A defects). More particularly, and except for a few intense spots obviously corresponding to aggregates, a quasi-full on and off switching of the TPL signal could be evidenced when going from an incident polarization parallel to perpendicular to the oily streaks [8]. TPL could thus prove that oily streaks enable the orientation of gold nanorods, opening interesting prospects in the field of directed nanoparticles assembly.

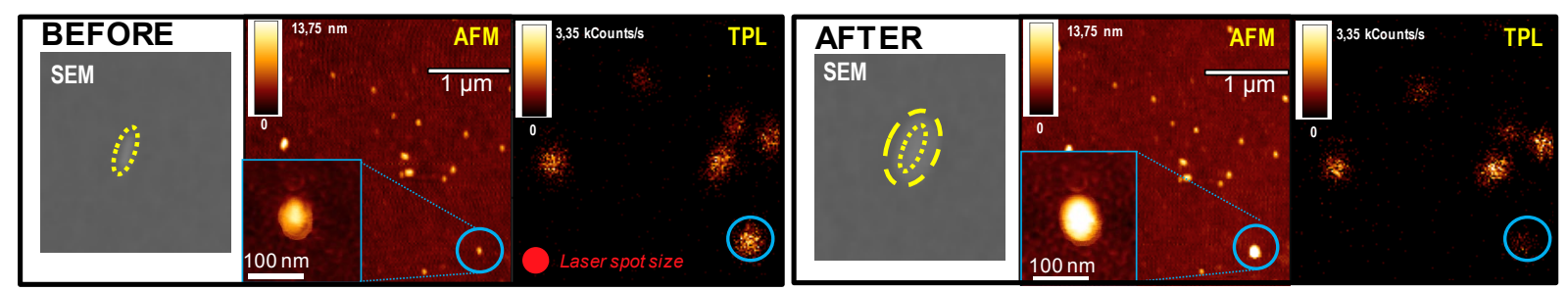

Figure 3. Correlated topography and TPL images of a sample of GNR immobilized onto an ITO coated glass cover slide, BEFORE and AFTER the "over-illumination" ( $\left.F=125 \mu \mathrm{J} . \mathrm{cm}^{-2}, \lambda=800 \mathrm{~nm}\right)$ of the blue circled GNR. High resolution tapping mode AFM scans of the blue circled GNR are displayed as insets of the topography scans, together with complementary SEM images.

The dependence of TPL on the excitation irradiance was also studied, previous reports stating changes in the luminescence emission spectrum in direct relation with the production of hot electrons and holes of higher energy $[15,16]$. As illustrated in Fig. 3, the illumination of single GNRs with increased energy density (from $125 \mu \mathrm{J} \cdot \mathrm{cm}^{-2}$ to $2.5 \mathrm{~mJ} \cdot \mathrm{cm}^{-2}$ ) leads however to a huge drop in their TPL intensity, even using energy densities well below the ones previously observed to induce photoinduced melting [3-6].

From theoretical simulations, we could show that femtosecond laser illumination with $125 \mu \mathrm{J} \cdot \mathrm{cm}^{-2}$ energy densities induce a temperature rise around $487 \mathrm{~K}$, below the melting of the particles, but high enough to enable gold atom spreading away from the GNR tip resulting in changes in the GNR plasmonic properties and thus TPL [7]. The shift of the rod resonance following reshaping leads to reduced absorption which acts as a feedback to stabilize the GNR shape and size. Conversely, the continuous matching of the shifted resonance wavelength offers a way to precisely reshape the GNR under illumination. This opens new prospects for the controlled photo-induced modification of single plasmonic particles using TPL.

\section{CONCLUSIONS}

The large two-photon luminescence of gold nanorods was shown to result from a "double-antenna" effect resulting from their two different localized surface plasmon resonances. Excitation of a gold GNR at its longitudinal plasmon frequency first lead to increased absorption (through strong local electromagnetic fields) and thus increased production of electron-hole pairs. Enhanced radiative recombination of these hot carriers is then observed through an antenna effect at the GNR transverse plasmon resonance [6], as it was also recently reported in the literature in the case of one photon luminescence [17]. A similar behaviour can also be evidenced 
in the case of colloidal gold nanobipyramids exhibiting non only dipolar but also higher order plasmon resonances (article in preparation). Our aim is now to determine the exact parameters enabling to control and optimize the radiative electron-hole recombination for a given localized plasmon resonance.

TPL was moreover demonstrated as a highly sensitive technique enabling to detect nanoparticles organization or reshaping. TPL was especially shown to evidence photo-induced morphological changes that happen to be hardly impossible to determine using common imaging methods such as AFM or SEM [7].

Above luminescence we will finally discuss other processes originating from plasmon relaxation, such as hot charges induced photophysics and photochemistry.

\section{ACKNOWLEDGEMENTS}

This work was supported by the French National Research Agency (ANR Blanc project HAPPLE).

\section{REFERENCES}

[1] S. Viarbitskaya, A. Teulle, R. Marty, J. Sharma, C. Girard, A. Arbouet, and E. Dujardin: Tailoring and imaging the plasmonic local density of states in crystalline nanoprisms, Nature Mat., vol. 12, no. 5, p. 426, 2013.

[2] S. Mitiche, S. Marguet, F. Charra, and L., Douillard: Near-field localization of single Au cubes: A group theory description, J. Phys Chem C, vol. 121, no. 8, p. 4517, 2017.

[3] J. A. Schuller, E. S. Barnard, W. Cai, Y. C. Jun, J. S. White, and M. L. Brongersma: Plasmonics for extreme light concentration and manipulation, Nat. Mater., vol. 9, p. 193-204, 2010.

[4] A. Mooradian: Photoluminescence of metals, Phys. Rev. Lett., vol. 22, 185-187, 1969

[5] L. Roloff, P. Klemm, I. Gronwald, R. Huber, J. M. Lupton, and S. Bange: Light emission from gold nanoparticles under ultrafast near-infrared excitation: Thermal radiation, inelastic light scattering, or multiphoton luminescence?, Nanolett., vol. 17, p. 7914, 2017.

[6] C. Molinaro, Y. El Harfouch, E. Palleau, F. Eloi, S. Marguet, L. Douillard, F. Charra, and C. FioriniDebuisschert: Two-photon luminescence of single colloidal gold nanorods: Revealing the origin of plasmon relaxation in small nanocrystals, J. Phys. Chem. C, vol. 120, pp. 23136-23143, 2016.

[7] C. Molinaro, S. Marguet, L. Douillard, F. Charra, and C. Fiorini-Debuisschert: From plasmon-induced luminescence enhancement in gold nanorods to plasmon-induced luminescence turn-off: A way to control reshaping, Phys. Chem. Chem. Phys., vol. 20, p. 12295, 2018.

[8] B. Rožič, J. Fresnais, C. Molinaro, J. Calixte, U. Shivakumar, S. Lau-Truong, N. Felidj, T. Kraus, F. Charra, V. Dupuis, T. Hegmann, C. Fiorini-Debuisschert, B. Gallas, and E. Lacaze: Oriented gold nanorods and gold nanorod chains within smectic liquid crystal topological defects, ACS Nano, vol. 11, no. 7, pp. 6728-6738, 2017.

[9] J. Xiao and L. Qi: Surfactant-assisted, shape-controlled synthesis of gold nanocrystals, Nanoscale, vol. 3, p. 1383, 2011.

[10] T. K. Sau and C. J. Murphy: Seeded high yield synthesis of au nanorods in aqueous solution, Langmuir, vol. 20, p. 6414, 2004.

[11] X. Huang, S. Neretina, and M. A. El-Sayed: Gold nanorods: From synthesis and properties to biological and biomedical applications, Adv. Mat., vol. 21, p. 4880, 2009.

[12] U. Hohenester and A. Trugler: MNPBEM - A Matlab toolbox for the simulation of plasmonic nanoparticles, Computer Physics Communications, vol. 183, no. 2, pp. 370-381, 2012.

[13] P. Zijlstra, J. W. M. Chon, M. Gu: Five-dimensional optical recording mediated by surface plasmons in gold nanorods, Nature, vol. 459 , pp. 410-413, 2009.

[14] A. Horneber, K. Braun, J. Rogalski, P. Leiderer, A. J. Meixner, and D. Zhang: Nonlinear optical imaging of single plasmonic nanoparticles with $30 \mathrm{~nm}$ resolution, Phys. Chem. Chem. Phys., vol. 17, pp. 21288-21293, 2015.

[15] T. Haug, P. Klemm, S. Bange, J. M. Lupton: Hot-electron intraband luminescence from single hot spots in noble-metal nanoparticle films, Phys. Rev. Lett., vol. 115, no. 6, p. 067403, 2015.

[16] O. Demichel, M. Petit, S. Viarbitskaya, R. Méjardde, F. Fornel, E. Hertz, F. Billard, A. Bouhelier, and B. Cluzel: Dynamics, efficiency, and energy distribution of nonlinear plasmon-assisted generation of hot carriers, ACS Photonics, vol. 3, pp. 791-795, 2016.

[17] Y. Y. Cai, J. G. Liu, L. J. Tauzin, D. Huang, E. Sung, H. Zhang, A. Joplin, W. S. Chang, P. Nordlander, and S. Link: Photoluminescence of gold nanorods: Purcell effect enhanced emission from hot carriers, $A C S$ Nano, vol. 12, no. 2, pp. 976-985, 2018. 